\title{
Avaliação do conhecimento sobre a bioequivalência dos fármacos em uma Universidade particular.
}

\author{
Knowledge assessment of bioequivalence of drugs in a private university
}

\begin{abstract}
Aleff Ricardo Santos Mendes, Amanda Caroline de Souza Sales, Brenda Ferreira de Oliveira, Matheus José Ferreira Carvalho, Maury Luz Pereira, Thayná Millena Nunes França.
\end{abstract}

\begin{abstract}
Resumo: A bioequivalência é definida pela ausência de diferença significativa na velocidade e extensão pela qual a substância ativa, presente em equivalentes ou alternativas farmacêuticas torna-se disponível no local de ação, quando administrado na mesma dose molar e sob condições similares em um estudo apropriadamente desenhado. É de suma importância avaliar o conhecimento das pessoas acerca deste assunto, haja vista que, no cotidiano, a bioequivalência dos fármacos é ignorada. Grande parte dos tratamentos com fármacos, inclusive a antibioticoterapia, não têm resultados efetivos devido à falta de informações dos pacientes. Neste sentido, este trabalho avaliou o conhecimento de homens e mulheres (funcionários, acadêmicos, professores e técnicos) sobre a bioequivalência dos fármacos. De acordo com os resultados previamente apresentados é possível concluir que a maioria das pessoas não sabe diferenciar medicamentos de referência, genéricos e similares. Homens e mulheres apresentam dificuldades para diferenciar embalagem de medicamento similar e de referência. O número de homens que compra medicamentos pela "qualidade" é menor em relação ao número de mulheres. Haja vista que em grande parte das perguntas, mulheres tiveram mais resultados positivos em relação ao conhecimento da bioequivalência dos fármacos, enquanto os homens entrevistados apresentaram mais resultados negativos em relação ao conhecimento da bioequivalência dos fármacos.
\end{abstract}

Palavras-chave: Bioequivalência, Fármacos,Universidade Particular

Abstract: Bioequivalence is defined by the absence of significant difference in speed and extent by which the active substance present in equivalents or pharmaceutical alternatives becomes available at the site of action when given in the same molar dose and under similar conditions in a study appropriately drawn. It is extremely important to evaluate the knowledge of the people about this subject, since, in the daily life, the bioequivalence of the drugs is ignored. Most drug treatments, including antibiotic therapy, do not have effective results because of lack of patient information. In this sense, this study evaluated the knowledge of men and women (employees, academics, teachers and technicians) on the bioequivalence of drugs. According to the results previously presented it is possible to conclude that most people do not know how to differentiate reference, generic and similar medicines. Men and women find it difficult to differentiate similar and reference drug packaging. The number of men who purchase "quality" drugs is lower than the number of women. In the majority of the questions, women had more positive results in relation to the knowledge of the bioequivalence of the drugs, whereas the men interviewed presented more negative results in relation to the knowledge of the bioequivalence of the drugs.

Keywords: Bioequivalence, Drugs, Private University

Curso de Biomedicina, Universidade Ceuma, São Luís, Maranhão, Brasil. 


\section{Introdução}

\section{Segundo}

biodisponibilidade é definida como a velocidade e extensão pela qual a substância ativa é absorvida da forma farmacêutica que não pretendem liberar o fármaco na corrente sanguínea, a biodisponibilidade pode ser determinada por medidas que reflitam a velocidade a extensão pela qual 0 ativo torna-se disponível no local de ação.

A bioequivalência é definida pela ausência de diferença significativa na velocidade e extensão pela qual a substância ativa, presente em equivalentes ou alternativas farmacêuticas torna-se disponível no local de ação, quando administrado na mesma dose molar e sob condições similares em um estudo apropriadamente desenhado ${ }^{1}$.

De acordo com a Agência Européia para avaliação de Produtos Médicos $(E M E A)^{2}$, dois produtos são considerados como bioequivalentes quando estes forem equivalentes ou alternativas farmacêuticas, cujas biodisponibilidades, após administração da mesma dose molar forem de tal forma semelhantes, que garantam os mesmos efeitos em relação à eficácia e segurança.

Um estudo de bioequivalência tem por objetivo comparar as biodisponibilidades de dois medicamentos considerados equivalentes farmacêuticos ou alternativas farmacêuticas e que tenham sido administrados na mesma dose molar. Se nestas condições as biodisponibilidades de dois medicamentos forem consideradas similares, os seus efeitos, no que respeita à eficácia e segurança dos mesmos serão essencialmente os mesmos $^{3}$. Estes estudos são administrados com base nos protocolos da Agência Nacional de Vigilância Sanitária.

Atualmente, o Brasil coloca-se na decima posição no ranking dos maiores consumidores de medicamentos do mundo, movimentando aproximadamente 5 bilhões de dólares por ano. Além disso, é o primeiro colocado mundial no número de farmácias, com cerca de 50 mil lojas, correspondendo a 3,34 lojas para cada 10 mil habitantes ${ }^{4}$.

É de suma importância avaliar o conhecimento das pessoas acerca deste assunto, haja vista que, no cotidiano, a bioequivalência dos fármacos é ignorada. Grande parte dos tratamentos com fármacos, inclusive a antibioticoterapia, não têm resultados efetivos devido à falta de informações dos pacientes. Ao compreender critérios de escolha entre homens e mulheres (professores, funcionários, acadêmicos e técnicos) da Universidade Ceuma, pode-se obter resultados importantes para compreender a forma utilizada na escolha de um fármaco. Assim, é necessário esclarecer as dúvidas sobre o assunto para uma melhor compreensão do tema. Neste sentido, este trabalho avaliou o conhecimento de homens e mulheres (funcionários, acadêmicos, professores e técnicos) sobre a bioequivalência dos fármacos.

\section{Material e métodos}

\section{Tipo de Estudo}

Estudo do tipo qualiquantitativo, prospectivo, direto.

\section{Local de Coleta de Dados e Período de Estudo}

Os dados foram obtidos a partir de fichas investigativas aplicadas na Universidade Ceuma, no município de São Luís - MA, de homens e mulheres, (funcionários, professores, acadêmicos e técnicos) acerca de seu conhecimento sobre bioequivalência. As fichas investigativas foram processadas no período de Novembro de 2015. 


\section{Cálculo Amostral}

A amostra do estudo é do tipo qualiquantitativo, onde analisou-se um total de 140 fichas investigativas - sendo 120 utilizadas para reprodução e continuação da pesquisa, e 20 descartados - de homens e mulheres (funcionários, professores e acadêmicos e técnicos) a partir de 16 anos de idade, independente de sexo e origem. A pesquisa durou em média 30 dias.

\section{Critérios de Inclusão}

a) Todos os funcionários, acadêmicos e professores da Universidade Ceuma.

b) Todas as fichas investigativas respondidas corretamente.

\section{Critérios de Exclusão}

a) Fichas investigativas com campos de informação em branco ou parcialmente preenchidos;

b) Professores de farmacologia.

\section{Coleta de Dados}

A coleta de dados foi efetuada de forma aleatória nas fichas investigativas, enfatizando o conhecimento de homens e mulheres sobre a bioequivalência dos fármacos e visando investigar a preferência dos entrevistados entre marca, preço, qualidade e indicação.

Também foi utilizado um instrumento de coleta de dados elaborado especialmente para este estudo. Este documento é composto por várias variáveis (idade, sexo, origem, tipos de medicamento, preço, marca, qualidade, indicação e diferença entre os fármacos), onde englobam o conhecimento a respeito da bioequivalência dos fármacos.

\section{Análises Estatísticas}

Os dados foram avaliados estatisticamente por meio do teste Quiquadrado, com resultados em gráficos e tabelas de frequência e suas variáveis. $\mathrm{O}$ nível de significância para que seja considerada a hipótese de nulidade é de $95 \%$, ou seja, considerar-se-á como estatisticamente significante um valor de $\mathrm{p}<0,05$.

\section{Análise de variáveis epidemiológicas}

Sexo

Temos uma população com predominância de mulheres (64\%) (Figura 1).

\section{Setor de entrevistados}

Temos uma altíssima predominância de acadêmicos (94\%). Uma menor participação de professores e funcionários e participação nula para alunos de nível técnico (Figura 2).

\section{Faixa Etária}

Grande maioria (72,67\%) dos indivíduos pesquisados que informaram sua faixa etária possui idade entre $18 \mathrm{e}$ 28 anos (Figura 3 ).

\section{Origem}

A grande maioria (72,5\%) dos indivíduos pesquisados é de origem autóctone (Figura 4). 


\section{Sexo}

$\square$ MASCULINO $\square$ FEMININO

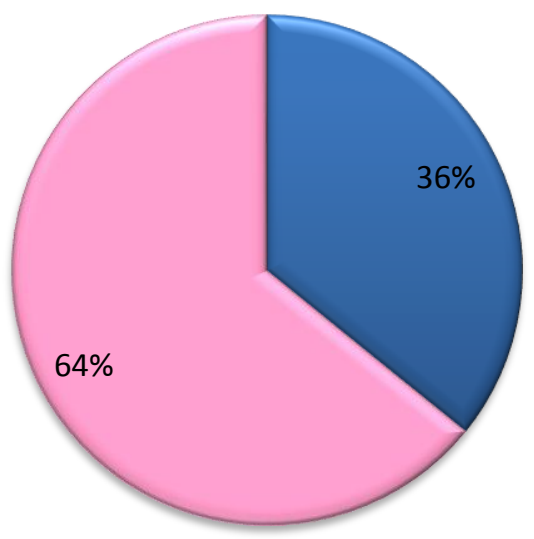

Figura 1. Distribuição de frequência do sexo do entrevistado

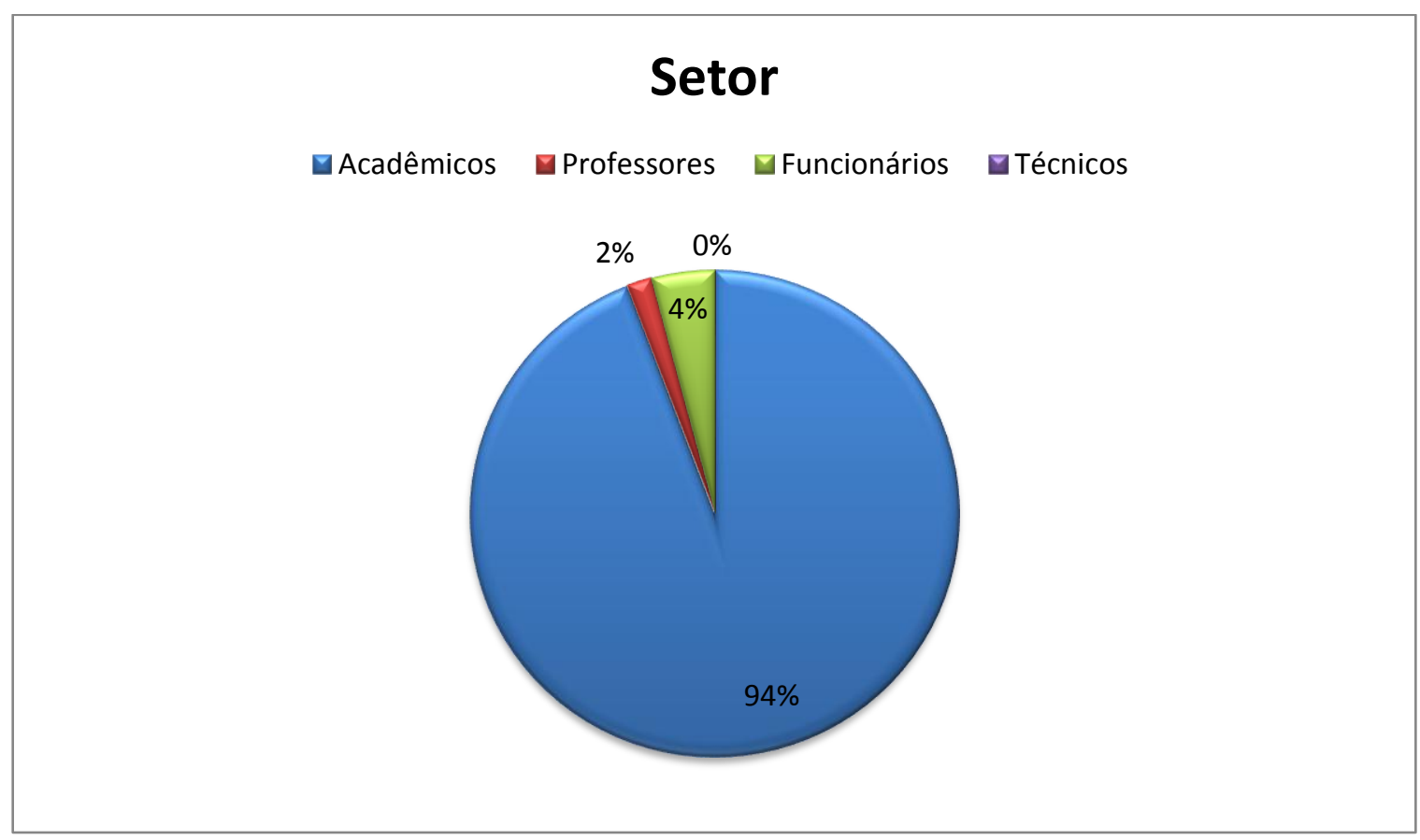

Figura 2. Distribuição de frequência do setor dentro da IES que o entrevistado ocupa. 


\section{Idade}

$\square<18$ anos $\square$ Entre 18 e 28 anos $\square>28$ anos

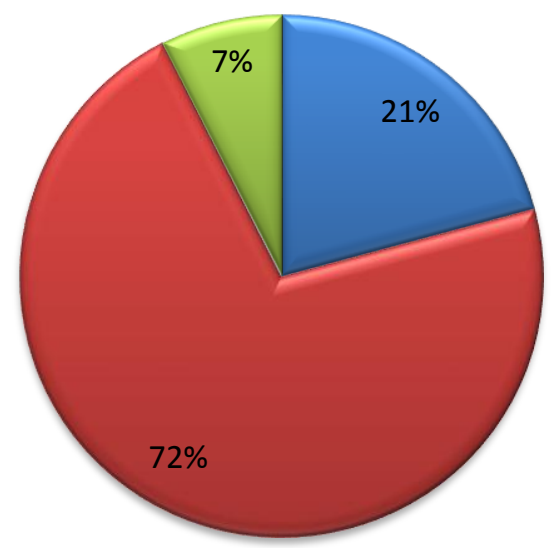

Figura 3. Distribuição de frequência da faixa etária do entrevistado

\section{Origem}

$\square$ Alóctone $\square$ Autóctone

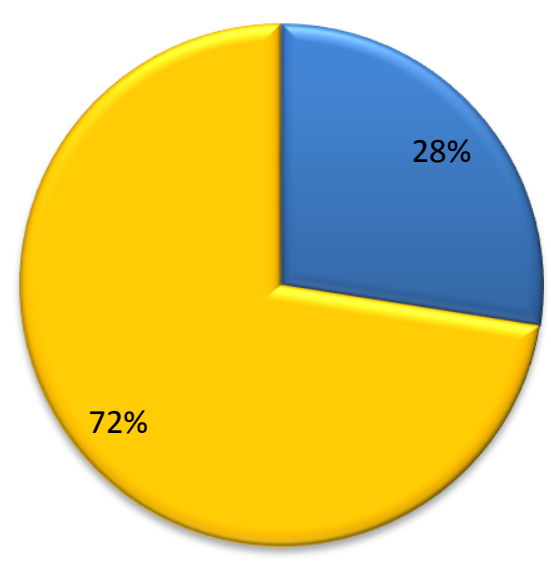

Figura 4. Distribuição de frequência da origem do entrevistado 


\section{Análise de variáveis quantitativas}

\section{"Você sabe o que é um medicamento genérico?"}

Identificamos que 0 grau de conhecimento das mulheres $(40,25 \%)$ em relação ao medicamento de referência é maior quando comparado aos homens $(30,23 \%)$ (Figura 5 ); onde $69,67 \%$ dos homens responderam que não sabem 0 que é um medicamento de referência e $49,75 \%$ das mulheres também responderam que não sabem (Figura 6)

\section{"Você sabe o que é um medicamento similar?"}

Identificamos que 0 grau de conhecimento das mulheres $(55,84 \%)$ em relação ao medicamento similar é maior quando comparado aos homens $(41,86 \%)$; onde $58,14 \%$ dos homens responderam que não sabem o que é um medicamento similar e $44,16 \%$ das mulheres também responderam que não sabem (Figura 7).

\section{"Quais os critérios utilizados quando você compra um medicamento?"}

Foi identificado que $47 \%$ das mulheres entrevistadas compram medicamento pelo preço, $15 \%$ compram pela marca, $25 \%$ compram por indicação e $13 \%$ compram pela qualidade (Figura 8).

Foi identificado que $47 \%$ dos homens entrevistados compram medicamento pelo preço. $16 \%$ compram pela marca, $30 \%$ compram por indicação e 7\% compram pela qualidade (Figura 9).

\section{"Opinião a respeito da embalagem de um fármaco e sua biodisponibilidade"}

Foi apresentada a imagem da embalagem do medicamento Buscopan ${ }^{\circledR}$ (Bultibrometo de escopolamina $10 \mathrm{mg} / \mathrm{ml}$ ) que é um medicamento de referência. Identificamos que $45,46 \%$ das mulheres e $39,54 \%$ dos homens responderam corretamente a questão proposta a respeito da biodisponibilidade do fármaco apresentado. $48,05 \%$ das mulheres e $55,81 \%$ dos homens não conseguiram diferenciar a embalagem de um fármaco similar e referência. $6,49 \%$ das mulheres e $4,65 \%$ dos homens não conseguiram diferencias a embalagem de um fármaco genérico e referência (Figura 10).

\section{Conclusão}

De acordo com os resultados previamente apresentados é possível concluir que a maioria das pessoas não sabe diferenciar medicamentos de referência, genéricos e similares. Homens e mulheres apresentam dificuldades para diferenciar embalagem de medicamento similar e de referência. O número de homens que compra medicamentos pela "qualidade" é menor em relação ao número de mulheres. Haja vista que em grande parte das perguntas, mulheres tiveram mais resultados positivos em relação ao conhecimento da bioequivalência dos fármacos, enquanto os homens entrevistados apresentaram mais resultados negativos em relação ao conhecimento da bioequivalência dos fármacos. 


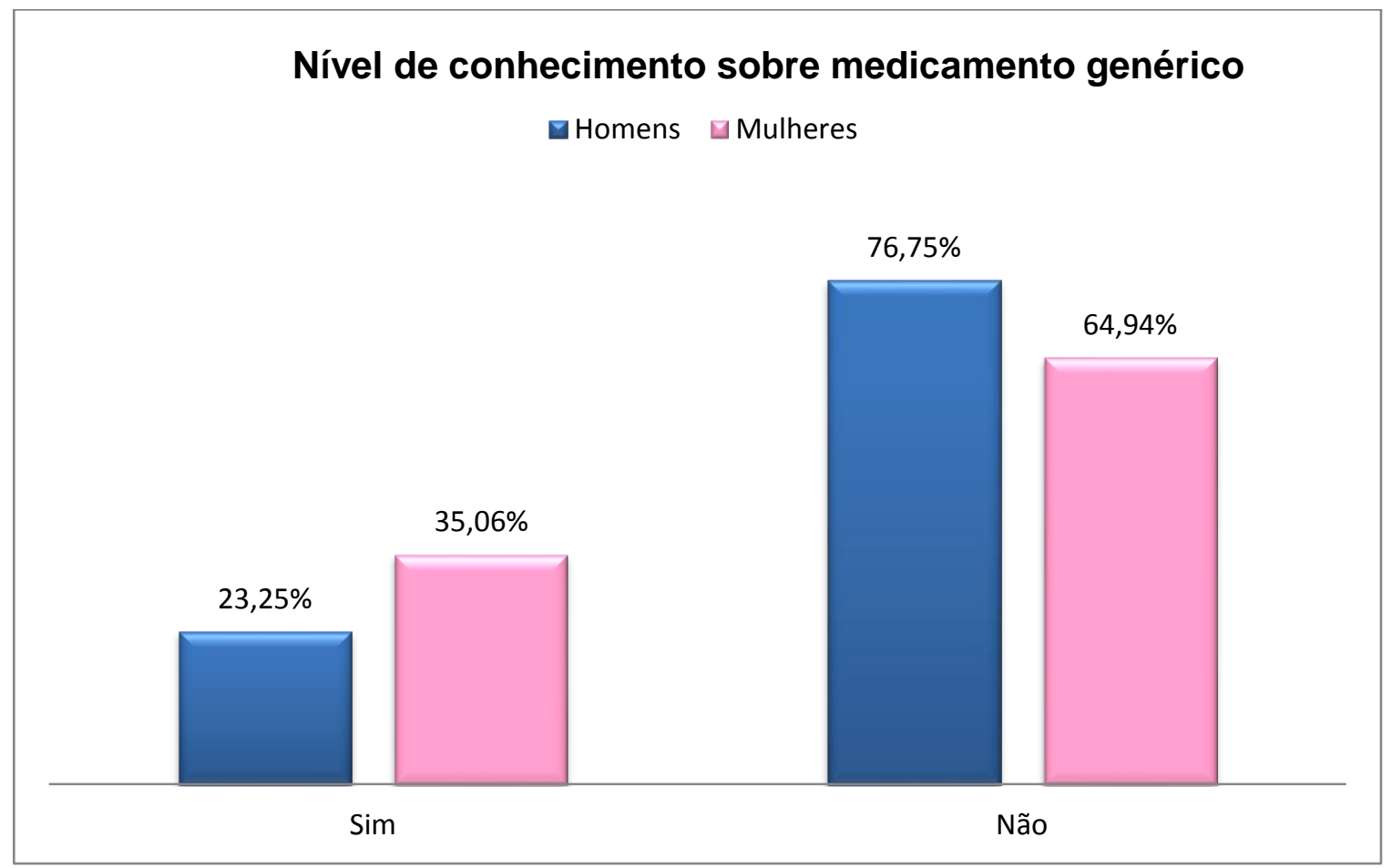

Figura 5. Distribuição de frequência sobre o nível de conhecimento sobre o medicamento genérico

Nível de conhecimento sobre medicamento de referência

- Homens $\square$ Mulheres

$69,67 \%$

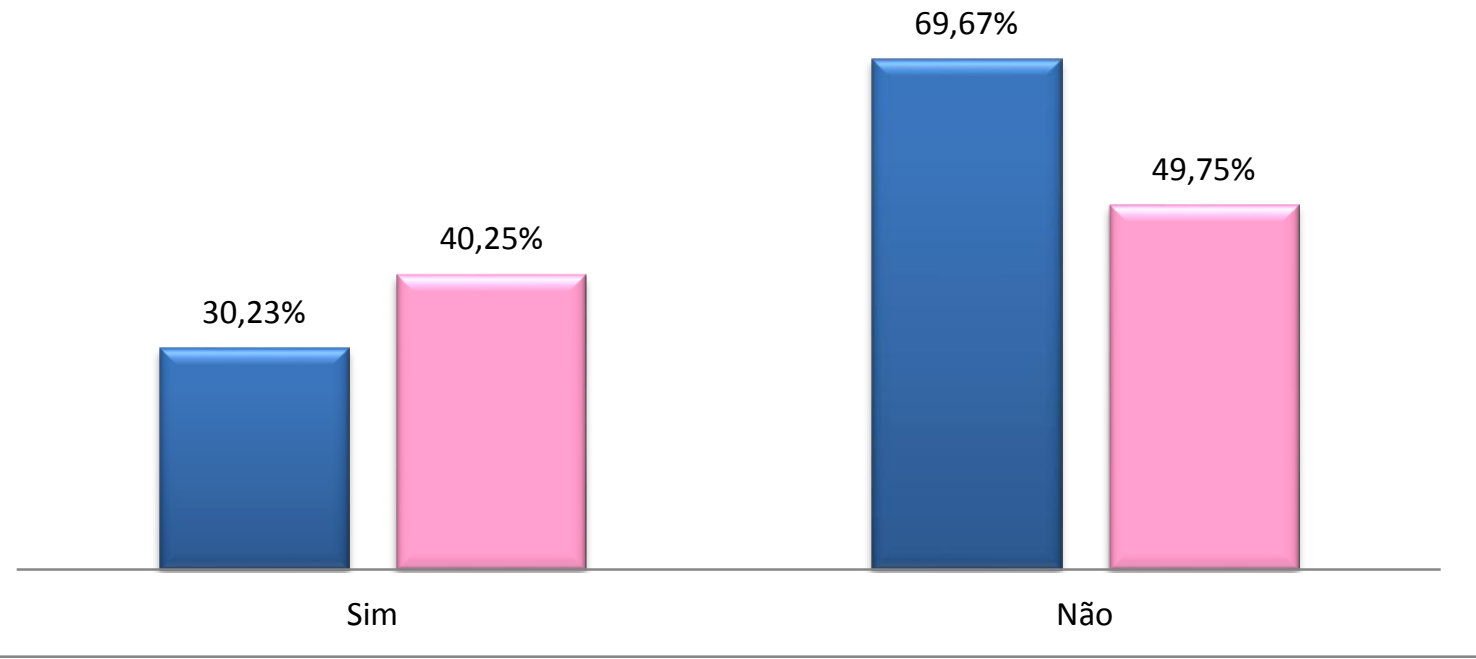

Figura 6. Distribuição de frequência sobre o nível de conhecimento sobre o medicamento de referência 


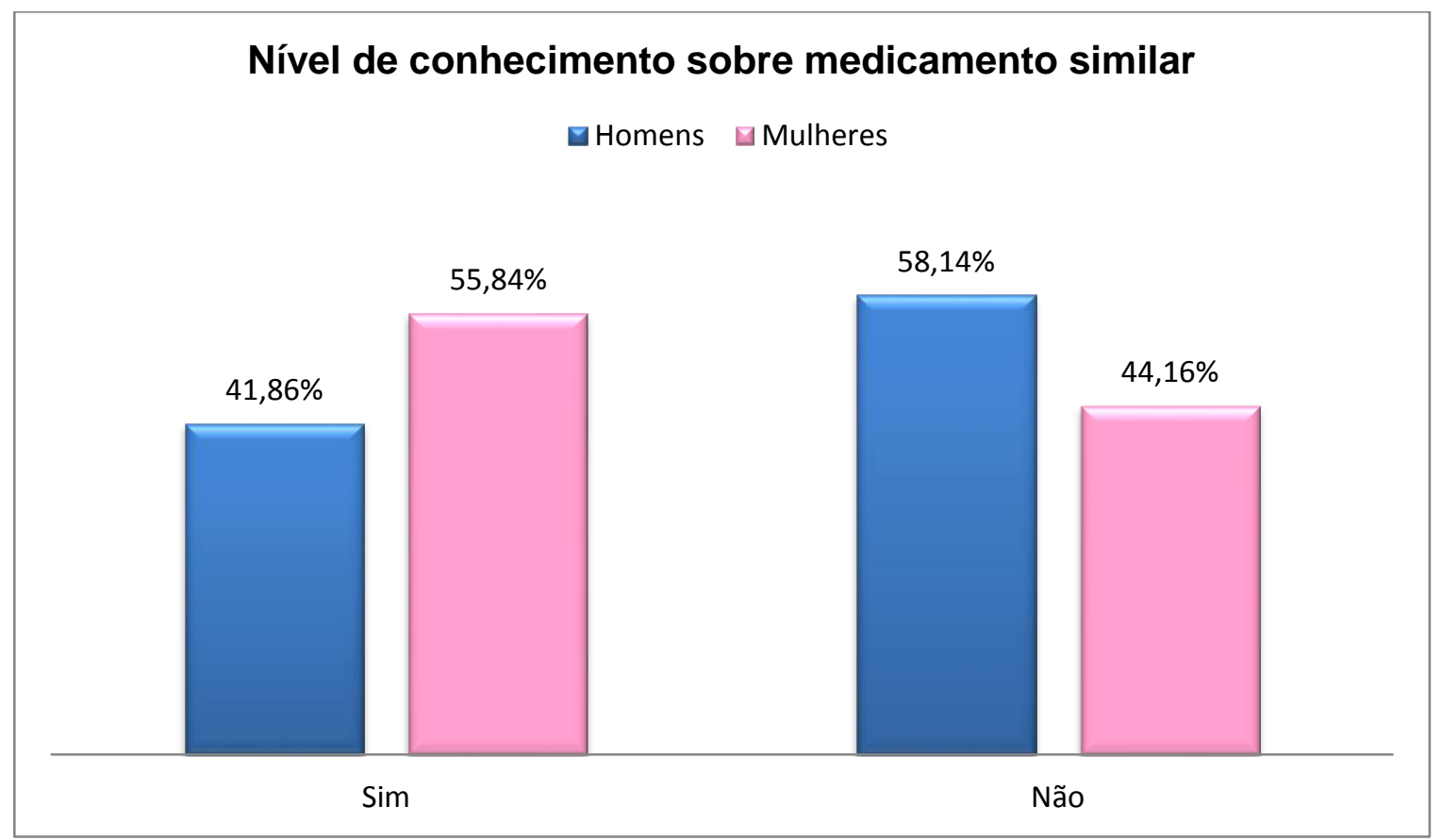

Figura 7. Distribuição de frequência sobre o nível de conhecimento sobre o medicamento similar

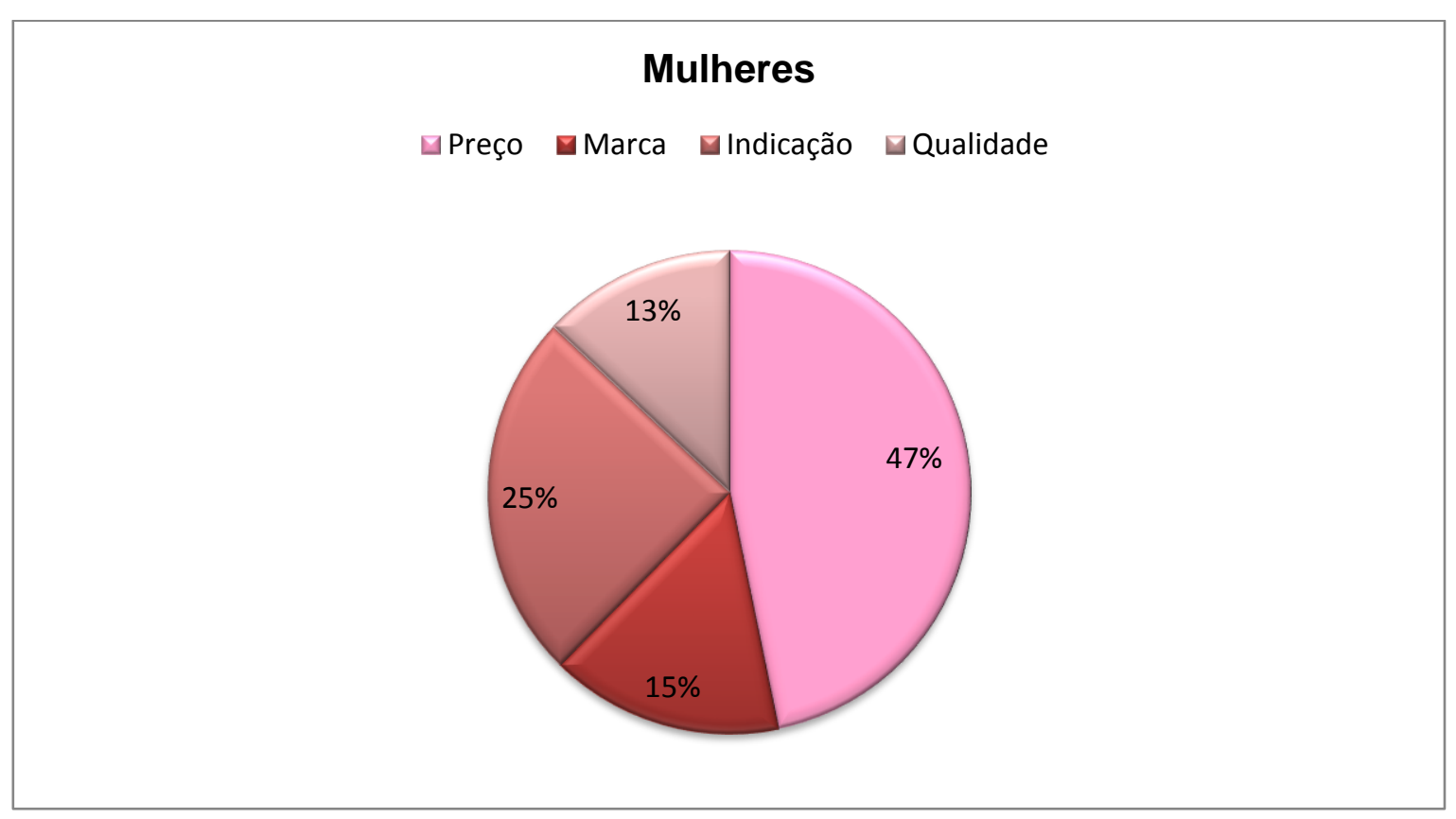

Figura 8. Critério das mulheres na compra de um medicamento 


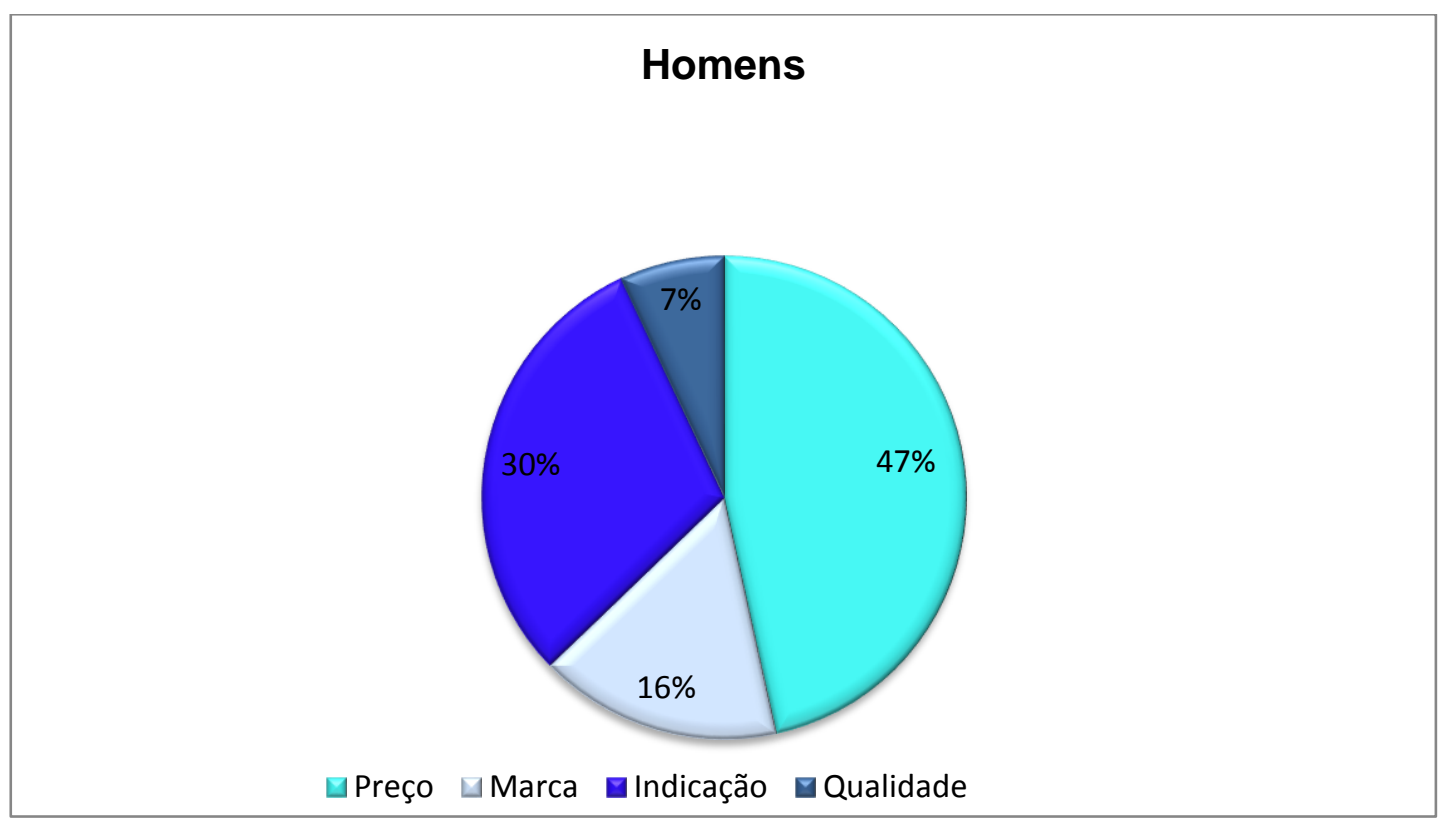

Figura 9. Critério dos homens na compra de um medicamento

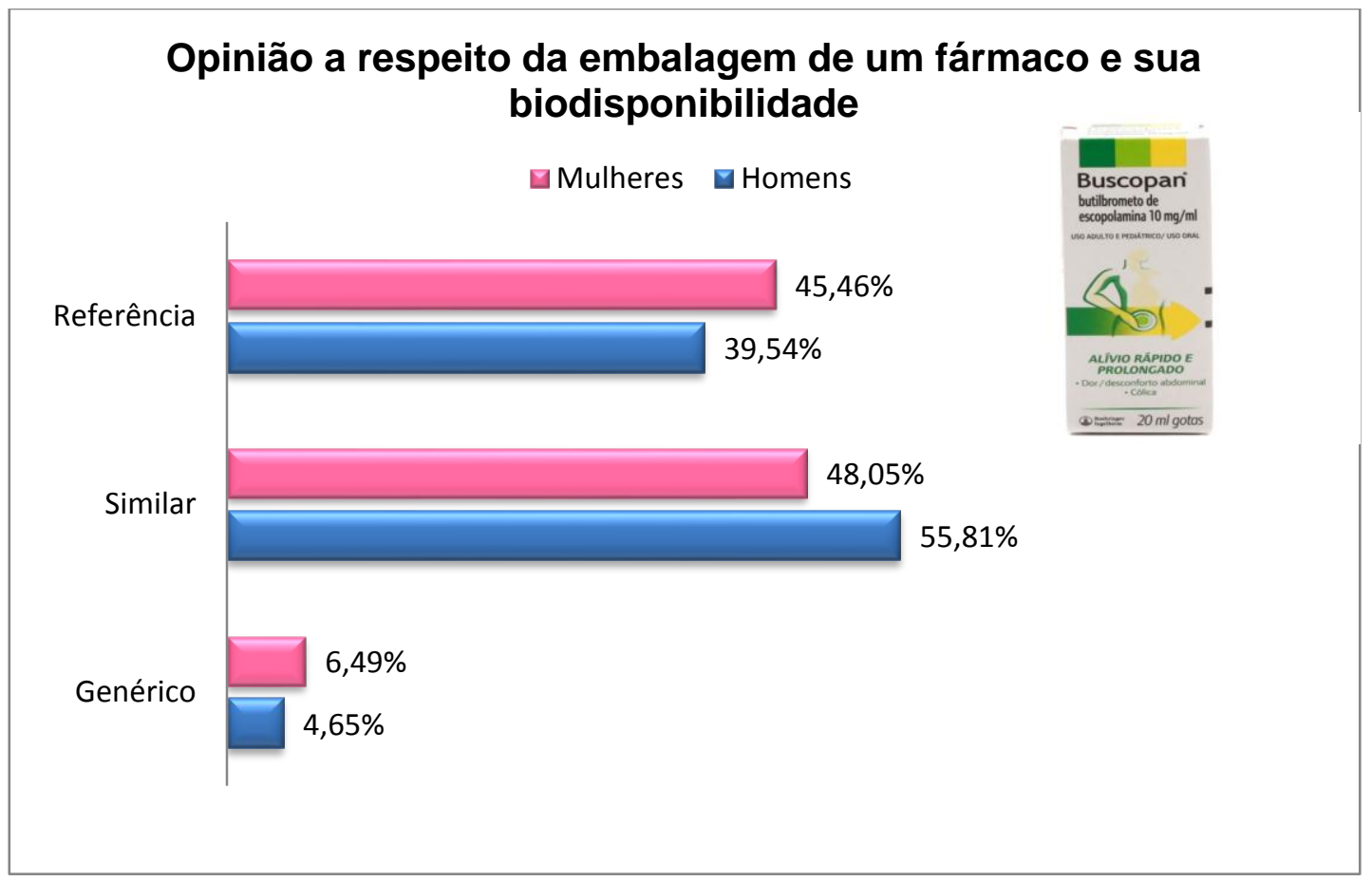

Figura 10. Opinião de homens e mulheres Critério dos homens na compra de um medicamento 


\section{Referências}

1. United States. Departament of Health and Human Services. Food and Drug Administration. Guidance for Industry: Bioanalytical Methot Validation. 2001. Disponível em: $<$ http://www.fda.gov/cder/Guindace/42 52fnl.pdf\#seach $=\% 22$ Food\%20and\%2 0Drug\%20Administration.\%20Guindan ce\%Validation $>$. Acesso em : 18 nov. 2015

2. European Agency For The Evaluation Of Medical Products. Evaluation Products. Note for guidance on the investigation of biovailabilty and bioequivalence. London: EMEA, 2001. 19p. (CPMP/EWP/QWP/1410/98).

3. Storpirtis, S.; Marcolongo, R.; Gasparotto, F.; Vilanova, C.M. A equivalência farmacêutica no contexto da intercambialidade entre medicamentos genéricos e de referência: bases técnicas e científica. In: BRASIL. Ministério da Saúde. Agência Nacional de Vigilância Sanitária. Anvisa Divulga. Artigos, Entrevistas e Relatórios. 2004. Disponível em: $<$ http://www.anvisa.gov.br/divulga/artig os/genericos referencia.pdf. $>$ Acesso em: 18 nov. 2015

4. Souza, J. Avalaiação das características fisico-quimicas, farmacocinéticas biodisponibilidade e da comprimidos. São Paulo, 2005. 290p. Tese de Doutorado - Faculdade de Ciências Farmacêuticas - Universidade de São Paulo.

5. Storpirtis, S. Biofarmacotécnica: fundamentos e biodisponiponibilidade, bioequivalência, dissolução e intercambialidade de medicamentos genéricos. São Paulo: s.n., 1999. 78p. 